See Article page 101.

\section{Commentary: All models are wrong, but some are useful}

\author{
Duy Cao Nguyen, MD, and Dawn S. Hui, MD
}

George E. P. Box is attributed with having said, "All models are wrong, but some are useful." The same can be said for 3-dimensional (3D) printing of the heart.

We develop models to improve our understanding of the real world. They simplify the complex. For cardiac diseases, these models began in medical school with gross anatomy, animal models, textbooks, diagrams, and images. All of this information is combined to develop a dynamic 3D model in our minds that allows cardiothoracic surgeons to understand the complex cardiovascular system, with further detail provided during each operation in an iterative manner. Improvements in resolution and virtual 3D reconstructions have significantly simplified this iterative model for surgeons. 3D printing has emerged from these as a new way to understand complex cardiac disease.

Henn and Mokadam ${ }^{1}$ review the history, current techniques, and applications for 3D printing for intracardiac surgery found in the literature. They provide a great primer of what $3 \mathrm{D}$ printing is, the process, and the current strengths and limitations of utilizing it in surgical training and decision making. Strengths include an accurate anatomic model to show anatomic relationships, predict the best place to incise/resect, and to accurately size implants. Limitations include the learning curve, the time to process/print, and cost.

Many groups are exploring the use of 3D printing in cardiac clinical decision making. ${ }^{2,3}$ Although Henn and Mokadam ${ }^{1}$ focus on the cardiac applications of 3D printing, there are already many general thoracic surgical applications of this

\footnotetext{
From the Department of Cardiothoracic Surgery, University of Texas Health Science Center at San Antonio, San Antonio, Tex.

Disclosures: The authors reported no conflicts of interest.

The Journal policy requires editors and reviewers to disclose conflicts of interest and to decline handling or reviewing manuscripts for which they may have a conflict of interest. The editors and reviewers of this article have no conflicts of interest.

Received for publication March 1, 2021; revisions received March 1, 2021; accepted for publication March 2, 2021; available ahead of print March 5, 2021.

Address for reprints: Dawn S. Hui, MD, Department of Cardiothoracic Surgery, University of Texas Health Science Center at San Antonio, 7703 Floyd Curl Dr, Suite 211L, San Antonio, TX 78258 (E-mail: huid@uthscsa.edu).

JTCVS Techniques 2021;9:109-10

2666-2507

Copyright (C) 2021 Published by Elsevier Inc. on behalf of The American Association for Thoracic Surgery. This is an open access article under the CC BY-NC-ND license (http://creativecommons.org/licenses/by-nc-nd/4.0/).

https://doi.org/10.1016/j.xjtc.2021.03.010
}

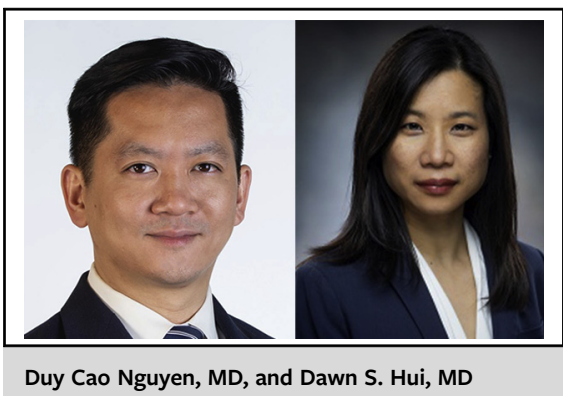

CENTRAL MESSAGE

3D printing offers potential to

understand, train, treat, and

innovate in all aspects of cardio-

thoracic surgery; applying these

developments will require mate-

rials, technology, funding, and

ingenuity.

technology, ${ }^{4}$ including resecting superior sulcus, chest wall, airway, and lung tumors through production of personalized models of the airway ${ }^{5}$ and pulmonary vasculature. ${ }^{6}$ The difference between a virtual 3D model and a 3D printed model has yet to be directly compared, but both will likely have roles in improving understanding and outcomes as accessibility and costs come down. Of note, a unique aspect of cardiac compared with general thoracic is the dynamic nature of the heart. Cardiac dimensions may be sensitive to factors such as preload and afterload, and this may influence the accuracy, fidelity, and precision of 3D printing. In these cases, the complex cannot and, perhaps should not, be simplified but rather modeled across the many various possibilities.

In parallel to augmentation of current techniques and understanding, it must be noted that from the industry side of things, 3D modeling and printing has been used in developing medical devices (from concepts to prototypes, including total artificial hearts ${ }^{7,8}$ ). Along with the very early development of bioprinting, ${ }^{9}$ 3D printing will certainly have an influence on cardiothoracic surgery and decision making. The potential for 3D printing is limitless, and we should all look forward to the innovations that this technology will provide.

\section{References}

1. Henn MC, Mokadam NA. JTVCS Techniques: Three-dimensional printing to plan intracardiac operations. J Thorac Cardiovasc Surg Tech. 2021;9:101-8.

2. Morkisz L, Gocol R, Deja M. Utilization of 3D printed heart model to tailor patch during surgical ventricular restoration. Available at: https://www.ctsnet.org/ 
article/utilization-3d-printed-heart-model-tailor-patch-during-surgical-ventricul ar-restoration. Accessed March 13, 2021.

3. Wang Y, Guo H, Wang S, Lai Y. Effectiveness of a patient-specific 3-dimensional printed model in septal myectomy of hypertrophic cardiomyopathy. Pak J Med Sci. 2020;36:1678-82.

4. Kwok JKS, Lau RWH, Zhao ZR, Yu PSY, Ho JYK, Chow SCY, et al. Multi-dimensional printing in thoracic surgery: current and future applications. J Thorac Dis. 2018;10(Suppl 6):S756-63.

5. Guibert N, Moreno B, Plat G, Didier A, Mazieres J, Hermant C. Stenting of complex malignant central-airway obstruction guided by a three-dimensional printed model of the airways. Ann Thorac Surg. 2017;103:e357-9.
6. Kurenov SN, Ionita C, Sammons D, Demmy TL. Three-dimensional printing to facilitate anatomic study, device development, simulation, and planning in thoracic surgery. J Thorac Cardiovasc Surg. 2015;149:973-9.e1.

7. Emmanuel S, Watson A, Connellan M, Granger E, Jansz P, Timms D, et al. First in man anatomical fitting study of the BiVACOR total artificial heart. J Heart Lung Transplant. 2020;39:4.

8. Bregar B. Plastics and the artificial heart. Available at: https://www.plasticsnews. com/article/20170706/NEWS/170709964/plastics-and-the-artificial-heart. Accessed March 13, 2021.

9. Dey M, Ozbolat IT. 3D bioprinting of cells, tissues and organs. Sci Rep. 2020;10: 14023. 